\title{
Movements, Literature, Aesthetics - Reflections on Dalit Ideology
}

\section{OPEN ACCESS}

Volume: 7

Issue: 2

Month: October

Year: 2019

P-ISSN: 2321-788X

E-ISSN: 2582-0397

Received: 17.08.2019

Accepted: 29.08.2019

Published: 01.10.2019

Citation:

Sindhu, A. "Movements, Literature, Aesthetics

- Reflections on Dalit Ideology." Shanlax International Journal of Arts, Science and Humanities, vol. 7, no. 2, 2019, pp. 55-58.

DOI: https://doi.org/10.34293/ sijash.v7i2.645

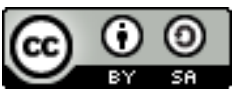

This work is licensed under a Creative Commons Attribution-ShareAlike 4.0 International License

\section{A. Sindhu}

Assistant Professor of Hindi, Payyanur College, Payyanur, Kerala, India

\section{Abstract}

This article describes the movements, literature and aesthetics of Dalit ideology in general. The codification of different angles and posititions in Dalit perspectives done here indicates a brief history and development of Dalit thought. The relation between the aesthetics and activism is visible here. Writing itself becomes not only mere an experience but also shifts as a tool for social change. So it exceeds the so called consciousness of aesthetics. Also it is a shift in social, cultural and political thinking of mankind. It attacks the development therories of modern man and civilization. In general Dalit aesthetics deconstruct the binary thinking and rationality of modernity. NatureCulture binary and its cultural boundaries became problematic here.

Keywords: आन्दोलन, अंबेदकर, दलित, दलित अवबोध, दलित चिंतन, दलित बहुजन, दलित साहित्य, सौन्दर्यशास्त्र

आन्दोलन, साहित्य, सौन्दर्यशास्त्र - दलित चिंतन का वैचारिक पक्ष

आज साहित्य और समाज में सबसे अधिक चर्चित शब्द है दलित। दलित शब्द के अर्थ और उसके अर्थव्याप्ति को लेकर वाद विवाद हो रहे है। दलित कौन है या दलित शब्द के भीतर कौन कौन आते है यह चर्चा करने का विषय है। दलित शब्द का प्रयोग सबसे प्रथम मराठी भाषा में ही प्रयुक्त हुआ था। दलित शब्द का प्रयोग ज्योति रावु फुले ने ही किया था। उन्होंने दमित अर्थ में ही इस शब्द का प्रयोग किया था। लेकिन कुछ लोगों का मानना

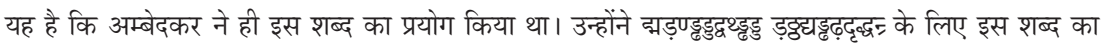
प्रयोग किया था। महाराष्ट्र में महर जाति को सूचित करने के लिए इस शब्द प्रयोग किया गया। १९३३ में गाँधीजी ने इसके लिए हरिजन शब्द का प्रयोग किया था।

\section{दलितः अर्थ और व्याप्ति}

दलित शब्द का वाचिक आर्थ है दमित लोग। "दलित शब्द दास, दस्यु, राक्षस, अशुद्ध, अछूत आदि शब्दों के बदले इस्तेमाल करते हैं।'(Massey: 1994:7)। 'दल' से ही दलित शब्द निकला है। दल का अर्थ है दमन किए गए, तोडे गए, रौन्दे गए आदि। विविध भाषाओं में दल का विविध अर्थ निकलते हैं। इन सभी अर्थों को देखा जाए तो दल शब्द का अर्थ दमित है।

१९७०-में 'दलित पान्तर ने ही दलित शब्द का प्रचार किया था। एक समुदाय से जोड़कर इस शब्द का प्रयोग तो ज्योतिबा फुले ने ही किया था'। उन्होंने अधकृत के उद्धार के लिए दलितोद्धार शब्द का प्रयोग किया। लेकिन आज यह दलित शब्द निम्न जाति के जातिस्वत्व बोध के परे ऐतिहासिक और सामाजिक अवबोध बन गए। बुनियादि तौर पर इस शब्द का प्रयोग वर्ण व्यवस्था और जाति व्यवस्था से जोड़कर प्रयोग किया था। आज यह सभी तरह से दमित लोगों के लिए प्रयुक्त किए जाने लगे। अस्मितादर्श के गंगाधर पाण्डव लिखते है कि - मेरे लिए दलित एक जाति नहीं अर्थिक और सामाजिक शोषण के शिकार है। वे ई-र पुनः जन्म और भेदभाव सिखाने वाले किसी धर्मग्रन्थ, स्वर्ग-नरक में वि-ास नहीं रखते। ये सब उन्हें गुलाम बनाते है। वे मानविकता पर वि-ास रखते है। दलित बदलाव और आंदेलन के प्रतिरूप है। (Etiest Zellior-1998:268) सभी तरह से दमित जनता को दलित कह सकते है। अधिकार के नीचे रहनेवाले यानी अधिकार के द्वारा दमित सभी लोग दलित है। लेकिन भारत में जाति के आधार पर दमित जनता को ही दलित कहते है विशेषकर हिन्दी साहित्य में।

भारत के संदर्भ में जाति ही शोषण की सबसे बड़ी बात रही थी। वर्णव्यवस्था और जाति व्यवस्था ने ही आम जनता का दमन किया था। इसलिए ही दलित शब्द का प्रयोग जाति के आधार पर दमित लोगों के लिए प्रयुक्त करते हैं। आदिवासी वर्ग, स्त्री आदि के लिए दलित शब्द का प्रयोग नहीं करते। स्वतंत्र भारत में सामाजिक अर्थिक रूप से शोषित और पीड़ित, जाति श्रेणी में सबसे निचले स्तर पर रहनेवाले लोगों के लिए अछूत जाति या अनुसूचित वर्ग शब्द का प्रयोग करने पर उनकी पीड़ाओं की तीव्रता व्यक्त नहीं होंगे। दूसरों द्वारा पीड़ित जनता है दलित। अछूत कहे गए भारत के सामाजिक श्रेणी में सबसे निचले स्तर में डाले गए रौन्दे गए जनता की संपूर्ण अवस्था को व्यक्त करने के लिए एक उचित शब्द के रूप में दलित शब्द का प्रयोग किया गया (हमीद;1998:32)। 
१९९३-में गाँधीजी ने दलित के लिए हरिजन शब्दका प्रयोग किया था। १९९९ के ब्रिटिश आक्ट में depressed class शब्द का प्रयोग किया गया। बाद में यह शब्द ड्टन्दड्दुद्धत्दृद्ध ड़ठह्मद्य शब्द का प्रयोग होने लगा। सैमण कम्मीशन ने इन्हें scheduled cast पुकारा। अंबेड़कर जब बौद्ध धर्म में चले गए तब ये लोग दड्टृद् डद्वडुडुत्म्मय जाने जाने लगे। लेकिन आज इन सभी शब्दों के बजाय दलित शब्द का प्रयोग होते है। गैल ओंवेद दलित शब्द को विशाल दृष्टि से देखते है। वे दलित पान्देस मानिफेस्टो को आधार में लेते हुए कहते हैं कि अनुसूचित जाति अनुसूचित वर्ग, neo buddist, मज़दूर वर्ग, भूरहित जनता, धर्म, राजनीति और अर्थ के आधार पर शोषित जनता आदि दलितों के भीतर आ जाते है। भारत में सबसे अधिक शोषित, अधिकारों से वंचित जनता अनुसूचित जाति व वर्ग है। समाज के सभी स्तर से हाशिए पर धकेले जाने वाले इन समूहों को दलित शब्दों के भीतर लाने पर इनमें नया बोध, प्रतिक्रिया, चलनात्मकता आदि आने लगे। कान्जा इलय्या इन्हें ‘दलित बहुजन' पुकारते है।

दलित एक जाति नहीं बल्कि अवबोध या पहचान है। दलित का अर्थ है आर्थिक संस्कृति सामाजिक और धार्मिक तौर पर दबाए जाने वाले शोषित जनता ।"(Arjun Dangle, poisoned bread, Bombay: Orient longman 1992, p.265) दलित शब्द ने शोषित जनता में अहं बोध पैदा किया। उनमें आत्मवि-ास पैदा कर स्वत्व बोध की स्थापना की। शोषण के विरुद्ध आवाज़ उठाने की शक्ति दी। इसलिए दलित शब्द एक आन्दोलन का नाम है। बाबु रावु बागुल लिखते हैं कि संपूर्ण परिवर्तन (आन्दोलन) का नाम दलित है। आन्दोलन का मूर्त रूप है यह। (Baburao Bagal, 'Dalit literature is human literature', Poisoned Bread, p.289) दलित शब्द भारतीय समाज के लिए हाशिएकृत अछूत जनता द्वारा दी गयी संभावना। सास्कृतिक आर्थिक सामाजिक व राजनैतिक ऐसा कहे भारतीय समाज के समूल परिवर्तन ही दलितों का लक्ष्य है। वे संगठित होकर सवर्ण सांस्कृतिक मूल्यों और ज्ञान व्यवस्था पर अधिष्ठित सामाज को बदलने की कोशिश कर रहे हैं। इसके लिए आज अनेक संस्थाएँ कार्यरत भी है। कुछ खास दर्शनों के आढ़ में आर्थिक सामाजिक और सांस्कृतिक रूप में शोषित जनजाति है दलित। वे अपनी मुक्ति के लिए एकजुड़ और एकमत होकर राष्ट्रीय और अंतरराष्ट्रीय स्तर पर आन्दोलन चला रहे है। इन आन्दोल से ही वे अपने को शोषण से मुक्त कराएगे। सभी भाषाओं में प्रयुक्त दलित शब्द आत्मशक्ति और धैर्य का शब्द है। इस शक्ति से वे स्वत्व निर्माण जैसे ऐतिहासिक प्रक्रिया में कार्यरत है।

\section{दलित आन्दोलन}

उन्नीसवीं शति समूचे भारत में नवजागरण का दौर रहा। नवजागरण ने कई सामाजिक आन्दोलन और संस्थाओं को जन्म दिया। फलस्वरूप देश भर में बहुत अधिक परिवर्तन आने लगे। पश्चिमी समाज से भारत का जो संबन्ध था वह सामाजिक व्यवस्था के संबन्ध में नए बोध पैदा किए। शिक्षा और नए काम काजी क्षेत्रों ने इस बोध को और भी विशाल बनाया। इस दृष्टि से जब समाज को देखने लगे तो सामाजिक परिवर्तन की माँग अंदर ही अंदर बुलन्द होने लगी। इस माँग ने देश में कई आँदोलनों को जन्म दिया। स्वाधीनता आन्दोलन से जुड़कर अनेक धार्मिक संस्थाओं की स्थापना हुई। प्रर्थनासमाज, आर्यसमाज, ब्रह्मसमाज, तियोसफिकल सोसाईटी जैसे संस्थाओं ने धर्म के भीतर स्थित समस्याओं में समूल परिवर्तन लाने के बजाय उनके आधुनिकीकरण में ही बल दिया। एक और ढंग से कहा जाए
तो ब्राह्मणाधिकार पर प्रश्न चिहन लगाने के बजाय ब्राह्मणवादी आशयों का आधुनिकीकरण ही इन संस्थाओं ने किया। अरविंद घोष, विवेकानन्दन जैसे सामाजिक नेताएँ भी सवर्ण ज्ञान व्यवस्था का निषेध करने में असमर्थ रहे।

अब्राह्मणों के जाति विरुद्ध आन्दोलनों की शुरुआत उन्नीसवीं शति के प्रथम दशक में ही होते है। पंजाब के आदि धर्म संस्था पश्चिम उत्तर प्रदेश के आदि हिन्दु संस्था, बंगाल के नामशूद्र संस्था, तमिलनाड के आदि द्रविड़ संस्था, महाराष्ट्रा के सत्य शोधक संस्था, केरल के श्री नारायण संस्था आदि कई संस्थाओं ने इस समय में जातिविरोध आन्दोलन की शुरुआत की। भक्ति आन्दोलन नवजागरण संस्थाएँ आदि ने ब्राह्मणवादी सिद्धान्तों पर विमर्शनात्मक दृष्टी डाली। ज्योतिबाफुले, रामस्वामी, श्रीनारायण गुरु और अय्यंकाली ने भौतिक और सामाजिक जीवन पर जोर देनेवाली अब्राह्मणिक दलित ज्ञान व्यवस्था को रूपायित करने की कोशिश की। इन कोशिशों को सैद्धान्तिक रूप देने का प्रयास अंबेदकर ने किया।

फुले के राय में निरक्षरता से ही सामाजिक असमत्व की शुरुआत होती है। इसलिए सत्यशोधक संस्था के जरिए उन्होंने आम जनता को शिक्षा देने का प्रयास किया। साथ ही साथ सवर्णांधिकार का प्रतिरोध किया। श्रीनारायणगुरु, अय्यंकाली दोनों ने सवर्ण आर्य समाज संकल्पना के बदले अब्राह्मणिक समाज की स्थापना की कोशिश की।

स्वतंत्रता और समता पर अधिष्ठित व्यक्ति संकल्पना को समाज के सामने रखते हुए अंबेदकर आए। वे भारतीय जाति व्यवस्था के प्रतिरोध के लिए पश्चिमी लिबरल मूल्यों को स्वीकारा। जाति को अस्वीकारते हुए उन्होंने कहा कि जाति व्यवस्था को तोड़े बिना छुआछूत का उन्मूलन संभव नहीं छुआछूत और जाति दो भिन्न भिन्न बातें नहीं। दोनों एक साथ जुड़े है। इसको अलग नहीं कर सकते। दोनों को एक साथ ही मिटा पाएगे। (अंबेदकर vol.४, १९९) अंबेदकर अपनी वेश भूषा से भी आधुनिक के रूप में लोगों के सामने प्रस्तुत हुआ न कि गाँधी के समान सन्यानी के रूप में । उन्होंने समझा कि समाज व्यवस्था के समूल परिवर्तन से ही दलितों का उद्धार संभव है। शिक्षा को उन्होंने एक सही विकल्प के रूप में स्वीकारा। इसके लिए १९४५ में मुंबई में सिद्धार्थ कॉलेज और मिलिन्द कॉलेज की स्थापना की। वर्ग नहीं जाति ही भारत की सामाजिक व्यवस्था में सबसे बड़ी बात है। धर्म के साथ जुड़े आचार-अनुष्ठान कानून ही जाति को बनाए रखते हैं। इसलिए उन्होंने हिन्दू धर्म को अस्वीकारा। अंबेदकर के संबन्ध में गैल ओमवेद लिखते हैं कि एक धर्म के रूप में हिन्दू धर्म को अस्वीकारे बिना जाति में निहित हीनताओं का उन्मूलन संभव नहीं। इसलिए एक बदल धर्म के रूप में उन्होंने बुद्ध धर्म को स्वीकारा ।"(२९९४:२२४-२५७)

राष्ट्र में अपने अस्तित्व के संबन्ध में दलित जनता अंबेदकर के समय से लेकर चर्चा करते है। संगठित शक्ति राजनीतिक अधिकार के संबन्ध में अंबेदकर की अवधारणाएँ दलित जनता में नए बोध पैदा किए। अमेरिका के ब्लाक पान्देर संस्था और साहित्य ने दलित पान्तेर को प्रचोदित किया। दलित स्वत्व बोध को व्यापक बनाने में ये बहुत अधिक सहायक हुए।

\section{दलित साहित्य}

दलित आन्दोलन का हिस्सा है दलित साहित्य। यह तो मान्य है कि समाज में जब कोई परिवर्तन या आन्दोलन चल रहे है तब साहित्य उससे अछूता नहीं रह सकते। साहित्य नए समाज के निर्माताओं में एक है। दलित साहित्य साहित्य के इस मान्यता से एक कदम ऊपर है। दलित साहित्य सामाजिक परिवर्तन को लिखनेवाले या सामाजिक आन्दोलन से प्रेरित 
साहित्य नहीं। जिस प्रकार दलित आन्दोलन या दलित मुक्ति संस्थाएँ दलित मुक्ति के लिए कार्यरत है उसी प्रकार कार्यरत एक क्षेत्र है दलित साहित्य। कहने का तात्पर्य यह है दलित साहित्य दलित मुक्ति जैसे लक्ष्य को सामने रखकर लिखे जानेवाले साहित्य है।

भारत के दबाए गए लोगों की जिन्दगी, उनके करुणादायक पीड़ाएँ,अपने हक के लिए चलाए गए आन्दोलन आदि से प्रेरित होकर दलित साहित्य का रूपायन हुआ। इसलिए दलित साहित्य विद्रोह का साहित्य है। सामाजिक परिवर्तन ही इस साहित्य का लक्ष्य है।

शिक्षा का प्रचार, आन्दोलन का दबाव और प्राप्त परिस्थिति के विरुद्ध संघर्ष के तहत दलित युवकों ने प्रस्थापित विषम समाज रचना के प्रति होनेवाला 'सन्ताप' और 'क्रोध' अपनी कलम से अभिव्यक्त किया। इसी लेखन को 'दलित साहित्य' के नाम से पहचाना जाने लगा।'(दलित साहित्य वेदना और विद्रोह-पृ. ४२) अपने ऊपर लादी गयी अमानवीय व्यवस्था के विरुद्ध ही दलित साहित्य का उदय हुआ।

१९५४ से लेकर ही 'दलित साहित्य' शब्द का प्रयोग हुआ। (Daya Pawas, History of Dalit literatureSouvenir, p-8) इससे पहले दलित साहित्य शब्द का प्रयोग तो नहीं हुआ था। फिर भी साहित्य में दलितों का जीवन कभी कभी विषय बने। १९५८ मुंबई में चले दलित साहित्य सम्मेलन ने एक अलग अस्तित्व के रूप में दलितों द्वारा लिखे गए साहित्य और दलितों के संबन्ध में लिखे गए साहित्य को दलित साहित्य नाम से स्वीकारने का निश्चय किया। यहाँ दलित साहित्य के भीतर दलितों द्वारा लिखे गए साहित्य गैर दलितों द्वारा लिखे गए साहित्य ये दोनों साहित्य गिने जाते थे। दलितों को शक्तिहीन बनाने वाली सभी व्यवस्था के प्रति ये आवाज़ उड़ाती है। कल तक दबाए रखे शब्दों का, विचारों का, विकारों का, अभिलाषाओं का और दुखों का आविष्कार ही दलित साहित्य के माध्यम से हुआ। यह एक जन समाज की स्वाधीनता के लिए की जाने वाली सांस्कृतिक प्रक्रिया है। वह चाहे दलितों के हाथों से हो या गैर दलितों के हाथों से। जनार्दनन वाङमरे लिखते हैं कि "दलित साहित्य बदला लेने के लिए आत्वान करनेवाला साहित्य, नहीं न ही विद्वेष पैदा करनेवाले साहित्य। दलित साहित्य मानव समाज की मुक्ति का आह्वान देते हैं। यह ऐतिहासिक माँग है। इसालिए ही यह अलग स्वत्व और संज्ञा को स्वीकारा। (Black literature and Dalit literature in poisoned Bread, 312)

दलित साहित्य का संबन्ध अंबेदकर के दलित वाद से है। अंबेदकर की राय में सामाजिक और सांस्कृतिक स्तर पर से उच्च नीच भाव मिटाए बिना दलितों की मुक्ति संभव नहीं। इसलिए जाति का उन्मूलन आवश्यक है। शैक्षिक, आर्थिक, सामाजिक स्तर में उन्नति पाकर परिवार के भीतर से और मानसिक स्तर से भी जाति को मिटाने की आवश्यकता है। दलित साहित्य इन विचारों को अमल में लाने के लिए कटिबद्ध दिखाई देते हैं।

दलित साहित्य आन्दोलन की शुरुआत महाराष्ट्र में हुई। महाराष्ट्र के साहित्यकारों के प्रतिक्रियावादी स्वर के रूप में ही दलित साहित्य का उदय हुआ। १९२० के संदर्भ में ही अंबेड़कर ने 'मूकनायक' साप्ताहिक निकाला था। दलित शब्दों को व्यापक बनाने में यह मागसिन सफलता प्राप्त की। बाद में दलित रचनाएँ न छापने वाले पत्र पत्रिकाओं को जलाकर लिट्टि ल मागसीनों को निकालकर प्रथम दौर में मराठी दालित रचनाएँ निकाली। राजाधेल, नामदेव दसल जैसों की रचनाएँ ऐसे निकले रचनाएँहैं।

प्रथम कालीन जातिविरुद्ध साहित्य और समकालीन दलित साहित्य का अंतर कर्तृत्व के संबंध में आए अवबोध है। फुले और लिंबाले की दृष्टि को
परखते हुए प्रदीपन पाम्बिरीक्कृन्नु लिखते हैं कि फुले की दृष्टि समाज को एक हद तक सौहार्द की दृष्टि से देखने और उपस्थित व्यवस्था से सहयोग देनेवाले थे। लेकिन लिंबाले की पीढ़ी दलित स्वत्व को आत्मवि-ास के साथ सवर्णस्वत्व के बदले में रखते हैं।(पृ. १०४, दलित पठनम्: स्वत्वम संस्कारम् साहित्यम) दलित साहित्य दलितों की आवाज को समाज के सामने रखते हैं।

दलित साहित्य आज समूचे वि-T में बहुत अधिक चर्चित साहित्य है। दलित और गैर दलित कई साहित्यकार आज सक्रिय ढंग से लिख रहे हैं। दलित लेखन प्रक्रिया स्वाधीनता हासिल करने की एक सांस्कृतिक प्रक्रिया है। मानवाधिकार को बचाने का आन्दोलन है। छुआछूत को भारत के संविधान गुणा के रूप में स्वीकारा है। यह आज मूर्त रूप से समाज में एक हदतक दिखाई नहीं दे रहे है। यह मूर्त रूप में न दिखाई दे या न दे एक मानसिक प्रक्रिया के रूप में आज भी विद्यमान है। इस मानसिक प्रक्रिया में परिवर्तन लाने का यानी सामाजिक व्यवस्था में परिवर्तन लाने का प्रयास ही दलित साहित्य करते हैं।

दलित साहित्य के निकलने के बाद कई तरह के तर्क वितर्क साहित्य क्षेत्र में निकले। दलित होकर जन्मे लेखक ही दलित साहित्यकार है। गैर दलित दलित साहित्यकार नहीं बन सकते ऐसे वाद विवाद शुरुआत से लेकर आज तक चल रहे हैं। इन चर्चाओं से तीन मत प्रबलरूप में निकल रहे हैं। वे ऐसा हे- १. दलित द्वारा लिखे गए २. दलितों को संबन्ध में लिखे गए ३. दलितों के लिए दलितों द्वारा लिखे जानेवाले साहित्य। दलित जीवन पर कई साहित्य निकल रहे हैं। गैर दलितों द्वारा लिखे गए साहित्य है और दलितों द्वारा लिखे गए साहित्य भी। लेकिन दलितों के साहित्य को दलित साहित्य मानने वाले लोगों को गैर दलितों के साहित्य में दलितों के प्रति सहानुभूति ही साहित्य में दिखाई देते हैं ऐसा कहकर उन साहित्यों को अस्वीकारते है। लिंबाले की राय में दलितों का जीवन उनका अनुभूति न होने से उन जीवन का यथार्थ चित्रण दलित साहित्य ही कर सकते हैं। गैर दलितों के दलित साहित्य में केवल सहानुभूति और दया का भाव ही देख सकता है। (दलित साहित्य का सौन्दर्यशास्त्र-१९६) निश्चय ही लेखक का अनुभव महत्वपूर्ण है। लेकिन दलितों के रूप में जन्म लेने से कोई दलित साहित्यकार नहीं बन सकते। दलित अवबोध से युक्त साहित्य ही दलित साहित्य बन सकते हैं। दलित विषयों को लिखने पर भी अगर यह साहित्य सवर्ण युक्ति को स्वीकारते है तो इसे दलित साहित्य नहीं कह सकेंगे। तात्पर्य यह है कि दलित साहित्य दलित अवबोध से युक्त साहित्य है। प्रो. क्षीरसागर कहते हैं कि दलित साहित्य की कोई जरूरत नहीं है। उनक्के राय में दलित अवबोध ही दलित साहित्य का मापदण्ड है। उसमें निश्चय ही दलित जीवन चित्रित होना है। अंबेदकर के विचार ही दलित साहित्य की प्रेरणा है। (R.K. Kshira Sagar, Dalit Literature, Souvenir, p-70).

दलित अवबोध की सृष्टि है दलित साहित्य। दलित अवबोध से तात्पर्य मानव के रूप में हम किसी से पीछे नहीं, अपने ऊपर लादे गए निम्नजाति स्तर और छुआछूत के विरुद्ध समता, नीति आदि मूल्यों के माँग को दलित साहित्य आगे रखते है। समता पर अधिष्ठित स्वाधीनता ही दलित अवबोध है। इस अवबोध के साथ जब रचना करते हैं तब यह दलित साहित्य बन जाते हैं। शरतचन्द्र मुक्ता के राय में दलित साहित्य दलित अवबोध की सृष्टि करते हैं। एक दलित लेखक को दलित दृष्टिकोण की आवश्यकता है। इस दृष्टिकोण से जब रचना करते हैं तब महत्वपूर्ण दलित रचनाएँ निकलते हैं। (What is Dalit literature, Poisoned Bread-267) बात यह है कि दलितों द्वारा लिखने से साहित्य दलित साहित्य नहीं बनते। 


\section{दलित साहित्य का सौन्दर्यशास्त्र}

दलित साहित्य का एक अलग सौन्दर्य शास्त्र है। दलित साहित्य केवल एक साहित्य पद्धति नहीं बल्कि यह सामाजिक आन्दोलनों का हिस्सा है। इसालिए लावण्यात्मक सौन्दर्यानुभूति से भी अधिक यह सामाजिक जीवन से अधिक जुड़े हुए है। यह शोषण के आधार पर बने सभी सामाजिक श्रेणी को तोड़कर रखने का प्रयास करते हैं। "दलित लेखक सामाजिक सरोकार के दायित्व से लिखता है। उसके लेखन से कार्यकर्ता का जोश और निष्ठा अभिव्यक्त होती है। दलित लेखक आन्दोलन में सक्रिय कार्यकर्ता-कलाकार है। वह अपने साहित्य को आन्दोलन मानता है। उसके सरोकार समग्रब्रोकन मेन से है।" (लिंबाले, दलित साहित्यः वेदना और विद्रोह, पृ.४३) सामाजिक बदलाव के लिए लिखे जानेवाले साहित्य होने के नाते दलित साहित्य को प्रस्थापित साहित्य की दृष्टि से देखकर उनका आस्वादन नहीं कर सकते। अब तक पाए गए साहित्यानुभूति या साहित्य सौन्दर्य से भिन्न है दलित साहित्य का सौन्दर्यशास्त्र। यह सामाजिक व्यवस्था में ही परिवर्तन नहीं लाते बल्कि साहित्य को देखने की दृष्टि में भी बदलाव लाते हैं।

दलित साहित्य का लक्ष्य दलित समाज को अवगत कराना और गुलामी के विरुद्ध संघर्ष करने के लिए उन्हें तैयार करना है। साथ ही साथ यह अभिजात वर्ग और साहित्य को एक चेतावनी भी है। भोगे हुए यथार्थ का बयान करके उससे ऊर्जा पाकर समाज के समस्त क्षेत्रों में वे अधिकार स्थापित कर रहे हैं। वह अधिकार जो सैकड़ों सालों से उनके हाथों से छीन लिया गया था। इसलिए दलित साहित्य में आनन्द की सौन्दर्य की अपेक्षा उपयोगिता का सौन्दर्य अधिक दिखाई देते हैं। ये साहित्य मुख्यधारा साहित्य से बिलकुल भिन्न है। इसलिए मुख्यधारा साहित्य के सौन्दर्य की कसौटी में रखकर दालित साहित्य के सौन्दर्य को आँका नहीं जा सकता। लिंबाले के शब्दों में दलित साहित्य की सौन्दर्य चर्चा करनी हो तो दलित साहित्य की निर्मिति, भूमिका, परिभाषा, विशेषता , प्रेरणा, प्रवृत्ति, स्वरूप और प्रयोजन के संबन्ध में विचार करना आवश्यक होता है...।"( दलित साहित्य का सौन्दर्यशास्त्र, पृ. ३२)

अस्वीकृति और नकार का साहित्य है दालित साहित्य। यह प्रस्थापित परंपरा इतिहास समाज अर्थशास्त्र, राजनीती, ज्ञानव्यवस्था आदि को नकारते है। सवर्ण अधिकार को स्थापित करनेवाले इन अमानवीय व्यवस्थाओं को तोड़ मरोड़कर नई व्यवस्था की स्थापना को लक्ष्य के रूप में स्वीकार कर लिखे जानेवाले साहित्य में व्यक्ति संघर्ष भी सामाजिक संघर्ष है। इन साहित्यों का स्वरूप एक जैसा है। यह अनुभवों की एक झुरी है। अस्पृश्यों का अस्पृश्यता के कारण होनेवाले अनुभव एक जैसा है। इसलिए समाज शास्त्र की दृष्टि से दलित साहित्य को आँकना है। तभी इसका सही विमर्श संभव हो पाएगा।

दलित चेतना और दलित आन्दोलन के विकास के साथ दलित साहित्य भी विकसित हुए। मराठी समाज संगठन शिक्षा संघर्ष और साहित्य के साथ दलित आन्दोलन आगे बढ रहे थे तभी हिन्दी समाज में भी आत्म सम्मान स्वाभिमान और समाज में आमूल परिवर्तन जैसे मुद्दों को लेकर दलित साहित्य आन्दोलन आगे चला। मोहनदास नैमिशराय कहते हैं कि उत्तर में कबीर आये नानक आये रैदास आये पर ज्योतिबा फुले और बाबासाहब डॉ.अंबेदकर जैसे व्यक्तित्व न आए। यह हमारे लिए अफसोस की बात क्यों
नहीं होनी चाहिए। हमने तो ज्योति व फुले और बाबा साहेब का जन्म होते ही उन्हें अपना मान लिया था। उनके विचारों को आत्मसात किया था। हमें लगा था कि उन्होंने हमारे ही घर में जन्म लिया। मेरा घर, मेरी बस्ती, मेरा नगर ही क्यों संपूर्ण हिन्दी क्षेत्र में हमने क्रान्तिकारी आह्वान किया था। (दलित चेतनाःसोच, पृ.३५ नवलेखन प्रकाशन, हज़ारीबाग) दलित साहित्य का संपन्न क्षेत्र और उसकी शुरुआत तो मराठा में हुआ था। लेकिन इन मराठा क्षेत्र ने सपूर्ण देश को आंदोलित किया। इस आन्दोलन से आन्दोलित होकर हिन्दी साहित्य में सभी दलित साहित्य स्पष्ट रूप से विकसित हुए।

आज दलित साहित्य समूचे भारत में बहुत अधिक निकल रहे है। दलित और दलितेतर अनेक साहित्यकार दलित साहित्य के क्षेत्र में उभर कर आ भी रहे हैं। सैद्धान्तिक तौर पर इस विषय में चर्चा करनेवाले लेखकों में गैल ओमवेद, कान्जा इलय्या, मीरा नन्दा, डी.आर.नागराज आदि प्रमुख है।दलित विमर्श जैसे विमर्श पद्धति सशक्त रूप में साहित्य क्षेत्र में चल रहे हैं। सृजनात्मक साहित्य भी कहानी, उपन्यास, नाटक आदि बहुत अधिक निकल रहे है।

\section{ग्रन्थसूचि}

इश्ट उमाशंकर चौधरी, 2011, दलित विमश कुछ मुद्दे और सवाल, आधार प्रकाशन, हरियाना

इश्रट डाँ. एन.सिं हा , 2015, दलित चिन्तनः अनुभव और विचार, वाणी प्रकाशन, नई दिल्ली

इइट डाँ. प्रदीपन पान्पिकिक्कुन्तु, 2007, दलित पठनम्ः स्वत्वम् स्क्कारम् साहित्यम् (मलयालम्), केरल भाषा इन्ट्टिट्यूट, तिरुवनन्तपुरम्

इ४टडाँ. बी.आर.अंबद्कर, 1996-2002, कलक्टड वक्स, अंबद्कर फौन्ड्शन, नई दिल्ली

इयट शरणकुमार लिंबाले, 2014, दलित साहित्य का सौन्दर्यशास्त्र , वाणी प्रकाशन, नयी दिल्ली

इ६ट शरणकुमार लिंबाले, 2010, दलित साहित्य : वेदना और विद्रोह , वाणी प्रकाशन, नयी दिल्ली

झ्टहमीद चेन्दमंगलूर, 1998, 'दलित प्रस्थानम'(मलयालम्), मातृभूमी साप्ताहिक, कॉकिक्कॉट

Dangle, Arjun. Poisoned Bread, Orient Blackswan, Mumbai, 1994.

Guha, Ranajith. Selected Subaltern Studies, Oxford University Press, NewYork, 1983.

Ilaiah, Kncha, Why I am not a Hindu, Samya, Culkatta, 1997.

Massey, James. Roots: A Conscious History of Dalits, CISRS, Banglore, 1994.

Omved, Gail, Dalit Vision: The Anti-caste Movement and the Construction of an Indian Identity, Orient longman, Delhi, 1995,

Zelliot, Eleanor, From Untouchable to Dalit, Manohar Publisher, New Delhi, 1998.

\section{Author Details}

A. Sindhu, Assistant Professor of Hindi, Payyanur College, Payyanur, Kerala, India,

Email ID: pinarayisindhu@gmail.com. 